\title{
Les Performances Agro-Economiques De L'urée Super Granulé : Cas Du Riz Au Sénégal
}

\author{
Babacar Faye, \\ Sadibou Sow, \\ Mamadou Diouf Fall, \\ Institut Supérieur de Formation Agricole et Rurale, \\ Université Alioune Diop, Senegal \\ Bachir Wade, \\ Faculté des Sciences Economiques et de Gestion (FASEG), \\ Université Cheikh Anta DIOP de Dakar, Senegal
}

Doi:10.19044/esj.2020.v16n13p364 URL:http://dx.doi.org/10.19044/esj.2020.v16n13p364

\section{Resume}

Le riz est l'aliment de base des sénégalais. Cependant sa production nationale est inférieure à la demande. La production du riz donne des rendements de 7 à 8 t/ha dans la vallée du Fleuve Sénégal avec une utilisation de grandes quantités d'urée. Dans cette étude, nous comparons les performances agronomiques et économiques de l'application de l'urée suivant deux technologies : la technique du placement profond de l'urée et l'épandage à la volée de l'urée. Un dispositif expérimental en blocs complets randomisés est utilisé avec quatre niveaux de fertilisation (T1 : $113 \mathrm{~kg} /$ ha d'urée super granulé (USG) de $1,8 \mathrm{~g}$ soit $52 \mathrm{~kg} \mathrm{~N} / \mathrm{ha} ; \mathbf{T 2}: 170 \mathrm{~kg} /$ ha d'urée super granulé (USG) de $2,7 \mathrm{~g}$ soit $78 \mathrm{~kg} \mathrm{~N} /$ ha; $\mathbf{T 3}: 226 \mathrm{~kg} /$ ha d'urée super granulé (USG) de 3,6g (2 USG de 1,8g) soit $104 \mathrm{~kg} \mathrm{~N} /$ ha et T4:300 kg / ha d'urée ordinaire soit $138 \mathrm{~kg} \mathrm{~N} / \mathrm{ha}$.). Les résultats montrent que la technologie du placement profond de l'urée permet d'obtenir des rendements plus élevés $\left(\mathrm{T}_{3}: 7582\right.$ $\mathrm{kg} / \mathrm{ha} ; \mathrm{T}_{2}: 7082 \mathrm{~kg} / \mathrm{ha}$ et $\mathrm{T}_{1}: 5441 \mathrm{~kg} / \mathrm{ha}$ ) que celui enregistré avec l'épandage à la volée de l'urée T4 (5375 kg/ha). L'analyse économique a montré un taux marginal de rentabilité de $1063 \%$ en prenant comme référence la fertilisation actuellement pratiquée.

Mots-clés: Riz, performance, urée, rentabilité, Sénégal 


\title{
Agro-Economic Performance of Super Granulated Urea: Case Rice on Senegal
}

\author{
Babacar Faye, \\ Sadibou Sow, \\ Mamadou Diouf Fall, \\ Institut Supérieur de Formation Agricole et Rurale, \\ Université Alioune Diop, Senegal \\ Bachir Wade, \\ Faculté des Sciences Economiques et de Gestion (FASEG), \\ Université Cheikh Anta DIOP de Dakar, Senegal
}

\begin{abstract}
Rice is the staple food of Senegalese people. However, its domestic production is below demand. Rice production yields 7 to $8 \mathrm{t} / \mathrm{ha}$ in the Senegal River Valley with the use of large amounts of urea. In this study, we compare the agronomic and economic performance of urea application using two technologies: the technique of deep placement of urea and the application of urea on the fly. An experimental device in randomized complete blocks is used with four fertilization levels (T1: $113 \mathrm{~kg} / \mathrm{ha}$ of super granulated urea (USG) of $1.8 \mathrm{~g}$ or $52 \mathrm{~kg} \mathrm{~N} / \mathrm{ha}$; T2: $170 \mathrm{~kg} / \mathrm{ha}$ of super granulated urea (USG) of $2.7 \mathrm{~g}$ or $78 \mathrm{~kg} \mathrm{~N} / \mathrm{ha}$; T3: $226 \mathrm{~kg} / \mathrm{ha}$ of super granulated urea (USG) of $3.6 \mathrm{~g}$ (2 USG of $1.8 \mathrm{~g}$ ) or $104 \mathrm{~kg} \mathrm{~N} / \mathrm{ha}$ and T4: $300 \mathrm{~kg} /$ hectare of ordinary urea or $138 \mathrm{~kg}$ $\mathrm{N} / \mathrm{ha}$.). The results show that deep urea placement technology provides higher yields (T3: $7582 \mathrm{~kg} / \mathrm{ha} ; \mathrm{T} 2: 7082 \mathrm{~kg} / \mathrm{ha}$ and T1: $5441 \mathrm{~kg} / \mathrm{ha}$ ) than that recorded with the on-the-fly application of T4 urea $(5375 \mathrm{~kg} / \mathrm{ha})$. The economic analysis showed a marginal rate of return of $1063 \%$ using the current fertilization as a reference.
\end{abstract}

Keywords: Rice; performance; urea; profitability; Senegal

\section{Introduction}

Le riz cultivé dans plus de 100 pays est l'aliment de base pour plus de trois milliards de personnes dans le monde. Sa production (dont plus 90\% vient d'Asie) mondiale a atteint 778,6 millions de tonnes en 2018. La Chine occupait la première 
place de ce classement suivie par l'Inde, l'Indonésie et le Bangladesh (source agence ecofin $)^{19}$.

L'Afrique subsaharienne, avec une superficie de 23,6 millions $\mathrm{km}^{2} \mathrm{n}$ 'a produit que 21,5 millions de tonnes sur une superficie de 10 millions d'hectares en 2010 (IFDC, 2013).

En Afrique de l'Ouest, le Sénégal est le premier pays consommateur de riz per capita avec $90 \mathrm{~kg} / \mathrm{an}$. Les importations de riz s'élevaient en 2014 à 958437 tonnes soit 177 milliards 883 millions FCFA (ANSD, 2014) ${ }^{20}$ tandis que la production nationale de riz ne couvrait en moyenne que 20 à $30 \%$ de la demande nationale en 2010. De plus, la flambée du prix de riz dans les années 2000 du fait de l'insuffisance de la production mondiale notamment des pays asiatiques, principaux fournisseurs du Sénégal, a accentué les difficultés d'approvisionnement du riz au Sénégal. Cette dépendance de l'extérieur pour une denrée alimentaire de base aussi stratégique constitue une préoccupation majeure des pouvoirs publics qui ont créé le Programme National d'Autosuffisance en Riz (PNAR) en 2009, révisé en 2014 dans le cadre du Programme d'Accélération de la Cadence de l'Agriculture Sénégalaise. Cette politique vise à satisfaire la demande nationale en riz.

Les rendements moyens sont passés de 4,02 t/ha en 2000 à 5,55 t/ ha en 2005, pour atteindre 7,5 t / ha en contre saison chaude en 2008 (PNAR, 2009) grâce à l'utilisation de 250 à $300 \mathrm{~kg}$ d'urée / ha. L'augmentation des rendements de $31 \%$ en riziculture est attribuée, entre autres, à l'utilisation des engrais dont l'urée (Lacharme, 2001). L'azote stimule la croissance augmente le rendement et la qualité du grain (Africa Rice, 2011). En riziculture irriguée, l'épandage de l'urée à la volée est la méthode d'application la plus répandue aussi bien en Asie qu'en Afrique. Avec cette méthode, les pertes sont estimées à 65 et $70 \%$ (Bhardwaj et al., 1993).

Ces quantités importantes d'urée utilisées se justifient par la faiblesse des taux de recouvrement de l'azote en milieu paysan qui ne dépassent pas les $30 \%$, (Ndiaye et $a l ., 2010$ ). Les engrais en général et l'urée en particulier représentent environ $55 \%$ du coût de production du riz irrigué.

L'autosuffisance en riz du Sénégal et la compétitivité de la filière du riz local par rapport au riz importé passent nécessairement par la diminution des écarts entre les rendements potentiels et les rendements actuels. Cette baisse augmente le rendement et la production de riz mais réduit les coûts de production et augmente la durabilité ${ }^{21}$.

\footnotetext{
${ }^{19}$ http://www. Agenceecofin.Com. / céréales /1507-67859- la-production-mondiale-de-rizatteint-778-6-millions-de-tonnes-en-2018-fao.

${ }^{20}$ ANSD : Agence National de la Statistique et de la démographie

${ }^{21} \mathrm{http} / /$ www.fao.org/rice2004/fr/f-sheet/fiche5.pdf
} 
La technologie du placement profond de l'urée (PPU) consiste à enfouir des super granules d'urée à une profondeur de 7 à $10 \mathrm{~cm}$.

Des travaux réalisés au Vietnam, au Népal et au Bangladesh, ont montré que le placement profond de l'urée permet de diminuer considérablement les quantités d'urée utilisées sur le riz, d'améliorer de façon significative le taux de recouvrement de l'azote de $60 \%$, d'augmenter le rendement paddy de $25 \%$, tout en réduisant les pertes par volatilisation de $35 \%$ dans le cas de l'épandage, à seulement 4\% (Prasad et al., 1982 Bhardwaj \& Singh, 1993). Au Sénégal, des expérimentations réalisées par l'Institut Sénégalais de Recherches Agronomiques (ISRA) en milieu paysan ont montré une plus-value de 1,17 t/ha par rapport à l'épandage d'urée (Ndiaye et al., 2010). La technologie du placement profond d'urée a permis d'atteindre des pics de rendement de 8,6 à 10 t / ha (ISRA, 2011).

C'est dans ce cadre que nous menons notre recherche dont l'objectif général est de contribuer à l'amélioration de la productivité du riz. Il s'agit d'évaluer dans la région de Matam les performances: agronomiques de l'application de l'urée super granulée en riziculture irriguée et économiques de la technologie du placement profond d'urée.

L'article est constitué de deux sections: la Méthodologie et la Présentation des résultats et discussion.

\section{Méthodologie \\ I.1 Zone d'étude}

La zone d'étude est située dans la rive gauche du fleuve Sénégal. L'essai a été implanté en milieu paysan en hivernage dans le casier rizicole de Matam. Ce casier couvre une superficie de 287 ha de sol hollaldé et polarise 574 producteurs dans 4 villages pour 12 unités autonomes d'irrigation (UAI). Le climat est de type sahélien, chaud et sec, avec des températures annuelles variant de 16 à $30^{\circ} \mathrm{C}$ en saison froide et de 35 à $48^{\circ} \mathrm{C}$ en saison chaude. Les précipitations moyennes dépassent rarement $500 \mathrm{~mm} / \mathrm{an}$. Au cours de ces dix dernières années, la moyenne enregistrée est de $398 \mathrm{~mm}$. Il y a deux saisons de culture du riz : une saison humide ou hivernage caractérisée par des températures élevées en début de culture (juin - juillet) et des températures basses en fin de cycle (Novembre - Décembre) et une saison sèche chaude caractérisée par de basses températures en début de culture (Février) et des températures élevées en fin de cycle (mi-juin).

\section{I.2. Matériels}

La variété utilisée est le Sahel 177 qui appartient à l'espèce sativa, au Groupe Variétal indica crée par Africa Rice, en 1998, homologuée et vulgarisée en 2009. Ses caractéristiques agronomiques sont intéressantes :

- un cycle moyen végétatif de 122 jours du semis à la maturité,

- une tolerance aux insectes, 
- une résistance à la verse,

- un potentiel de rendement de 10 t/ha.

En outre, cette variété aromatique, avec ses caractéristiques organoleptiques, est très prisée des consommateurs sénégalais.

Le fertilisant utilisé est l'Urée Super Granulée (USG) qui est fabriquée, à partir de l'urée ordinaire, par une machine appelée granuleuse ou « briqueteuse ». La machine compresse l'urée pour obtenir des super granules dont le poids unitaire est en fonction des rouleaux à alvéoles calibrées $(1,8 \mathrm{~g}$ et $2,7 \mathrm{~g})$.

\section{I.3. Dispositif expérimental}

Un seul facteur (fertilisation azotée) est étudié dans un dispositif en blocs complets randomisés avec quatre niveaux (ou traitements) et quatre blocs ou répétitions. Les traitements sont :

T1 : $113 \mathrm{~kg} /$ ha d'urée super granulé (USG) de $1,8 \mathrm{~g}$ soit $52 \mathrm{~kg} \mathrm{~N} /$ ha ;

T2 : $170 \mathrm{~kg}$ / ha d'urée super granulé (USG) de $2,7 \mathrm{~g}$ soit $78 \mathrm{~kg} \mathrm{~N} / \mathrm{ha}$;

T3 : $226 \mathrm{~kg} /$ ha d'urée super granulé (USG) de 3,6g (2 USG de 1,8g) soit 104 $\mathrm{kg} \mathrm{N} / \mathrm{ha}$;

T4 : $300 \mathrm{~kg} /$ ha d'urée ordinaire soit $138 \mathrm{~kg} \mathrm{~N} /$ ha.

L'unité expérimentale est la parcelle élémentaire est un carré dont la superficie est de $16 \mathrm{~m}^{2}$

\section{I.4. Conditions expérimentales}

L'expérimentation a été faite durant la campagne 2014-2018.Nous avons utilisé $40 \mathrm{~kg}$ de semence pour la pépinière sur une superficie de 500 $\mathrm{m}^{2}$ qui permet le repiquage d'un hectare. Un travail du sol sous forme d'offsetage est réalisé sur l'ensemble de l'essai.

Le repiquage d'un plant par poquet a été fait avec des écartements de $20 \mathrm{~cm}$ entre les lignes et $20 \mathrm{~cm}$ sur la ligne à une profondeur de $3 \mathrm{~cm}$. La densité obtenue est de 250000 plantes / ha.

Une fumure de fond de 18-46-00 a été appliquée à la dose de $100 \mathrm{~kg} /$ ha sur toutes les parcelles deux jours après le repiquage. Au septième jour après repiquage, les super granules d'urée ont été enfouis à la main entre quatre poquets de riz pour les traitements $\mathrm{T} 1, \mathrm{~T} 2$ et $\mathrm{T} 3$ en prenant soin de fermer hermétiquement les trous. Pour le traitement T4, les $300 \mathrm{~kg}$ d'urée sont appliqués à la volée en deux fractions égales : $50 \%$ au début du tallage soit environ 14 jours après repiquage et $50 \%$ à l'initiation paniculaire (environ 43 jours après repiquage). Deux sarclo-binages manuels ont été faits en début du tallage et à l'initiation paniculaire.

Conformément au planning consensuel au sein du casier, les parcelles sont irriguées tous les quinze jours. Un traitement au Malathion a été effectué à la dose d'un litre par hectare au 55éme jour après repiquage à la suite d'une 
attaque de la mouche blanche (Bemisia tabaci). Pour estimer le rendement (paille et paddy), nous avons récolté, à l'aide d'une faucille, les plantes d'une placette de $1 \mathrm{~m}^{2}$ à l'intérieur de chaque parcelle pour minimiser l'effet bordure.

\section{I.5. Collecte des données}

A la maturité, un échantillon de 10 plantes matérialisées par des piquets distants de $0,5 \mathrm{~cm}$ le long de la diagonale d'une parcelle, nous avons compté le nombre de talles et de panicules par plante et mesuré la hauteur des plantes. Au total, 25 plantes entières (paille et grains paddy) ont été récoltées, séchées puis pesées.

Après battage et vannage, l'humidité des grains est mesurée avant leur pesage. Les rendements ont été évalués en tenant compte des ajustements au taux d'humidité standard de $14 \%$ selon la formule suivante:

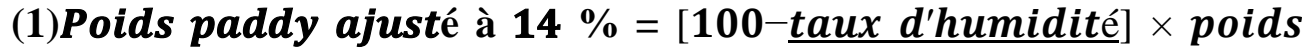
(kg) échantillon 100-14

Le rendement de riz paddy /ha de chaque parcelle élémentaire est donné par la formule suivante :

(2) Rendement $(\mathrm{kg} / \mathrm{ha})=\left[\begin{array}{l}\text { Poids paddy ajusté à } 14 \% \\ \hline\end{array}\right.$ $[(\boldsymbol{k g})]^{* 10000 \mathrm{~m} 2}$ Superficie unitaire en $\mathrm{m}^{2}$

L'indice de récolte est calculé en faisant le rapport entre le poids des grains et le poids de la paille avant de déterminer l'efficience de l'azote qui est le rapport entre la quantité de paddy par kg d'azote appliqué.

\section{I.6 Analyse économique}

Pour l'analyse économique, nous avons suivi les trois étapes suivantes : l'élaboration du budget partiel, l'analyse de la dominance et la détermination du taux marginal de rentabilité de Ndiaye et Sidibé (1999) qui ont utilisé cette méthode pour évaluer la rentabilité économique de la fumure minérale sur la culture du maïs dans la zone de Nioro au centre - sud du Sénégal.

\section{I.6.1 Budget partiel}

Nous avons élaboré un budget partiel de culture pour les différents traitements. La valeur de la production est égale au rendement de paddy exprimé en $\mathrm{kg}$ / ha multiplié par le prix du kg de paddy. Nous avons considéré le prix officiel du paddy fixé pendant la campagne d'hivernage 2015/2016 par les acteurs de la filière Riz. Les coûts variables sont obtenus en faisant la somme des coûts des intrants et des coûts opérationnels.

- Les intrants comprennent : la semence appliquée à la dose de $40 \mathrm{~kg}$ / ha en raison de 375 
FCFA / kg, l'engrais de fond Di Ammonium Phosphate (DAP) appliqué à la dose de

$100 \mathrm{~kg} / \mathrm{ha}$ au prix de $176 \mathrm{FCFA} / \mathrm{kg}$, l'urée ordinaire apportée à la dose de $300 \mathrm{~kg} /$ ha au prix de 159,5 FCFA / $\mathrm{kg}$, l'urée super granulée (USG) au prix de 175,5 FCFA / kg y compris le coût de la granulation qui est de 16 FCFA $/ \mathrm{kg}$, le sac de conditionnement du paddy est acheté à 100 FCFA dans le marché local, le Malathion est appliqué à la dose d'un litre par hectare au prix de 10000 FCFA le litre ;

- Les coûts opérationnels correspondent aux coûts de l'utilisation de la main d'œuvre

Exprimés en UTH.j ${ }^{-1}$ (Unité de travail d'un homme durant 8 heures par jour) pour la confection des diguettes, la gestion des irrigations, le repiquage en ligne, l'enfouissement des granulés, les épandages de DAP et d'urée ordinaire, le sarclage et le traitement phytosanitaire. Dans la zone, le coût de la maind'œuvre est fixé à 1500 FCFA/jour. Le coût du transport du paddy est estimé sur la base d'une charge de charrette à 3500FCFA la tonne.

Des coûts forfaitaires de 27000 FCFA / ha pour effectuer l'offsetage et $66000 \mathrm{FCFA} /$ ha pour réaliser les opérations mécaniques de récolte et de post- récolte ont été considérés. Dans le casier de Matam, des valeurs forfaitaires de $80000 \mathrm{FCFA} /$ ha pour le coût hydraulique et $5500 \mathrm{FCFA} / \mathrm{ha}$ pour la redevance de l'OMVS 22 ont été utilisées.

À partir du budget partiel, nous calculons le bénéfice net qui correspond au gain additionnel en faisant la différence entre la valeur de la production et les coûts variables.

( 3 ) bénéfice net $=$ valeur de la production - et coûts variables

\section{I.6.2. Analyse de dominance}

Elle a pour objectif de mesurer les performances d'une technologie ou plan de fumure à travers l'ampleur de son bénéfice net, compte tenu de son coût total de réalisation. Une technologie est considérée comme dominée s'il est possible de trouver une autre qui coûte moins chère tout en rapportant un bénéfice net plus élevé; dans le cas contraire, la technologie est dite supérieure. Si la technologie est dominée, le gain du rendement additionnel ne parvient pas à compenser les coûts additionnels engagés.

\section{I.6.3. Analyse marginale}

L'analyse marginale permet de quantifier l'ampleur relative des technologies qui présentent un intérêt économique déterminé par l'analyse de dominance. Les technologies qui représentent un intérêt économique pour le producteur sont les technologies supérieures dont le taux marginal de

${ }^{22}$ OMVS : Organisation pour la Mise en Valeur du fleuve Sénégal 
rentabilité selon l'option qui l'intéresse, traduit le comportement des bénéfices nets reliés à la technologie par rapport aux coûts engendrés en intrants et en main d'œuvre. Le bénéfice net marginal et les coûts variables marginaux sont calculés pour les options technologiques (les niveaux de fertilisation) supérieures et pour lesquelles les bénéfices nets et les coûts variables marginaux ainsi que le taux marginal de rentabilité sont calculés.

Les bénéfices nets marginaux sont déterminés de la façon suivante :

(4) Bénéfice Marginal de l'option technologique $X=$ Bénéfice Net de l'option technologique $(X)$ - Bénéfice Net de l'option technologique de référence $(Y)$; Les coûts variables marginaux sont déterminés de la façon suivante :

(5) Coût Marginal de l'option technologique $X=$ Coût Variable de l'option technologique $X$ - Coût variable de l'option technologique de référence $Y$.

Les taux marginaux de rentabilité (TMR) comparent l'augmentation des charges variables qu'entraine le passage d'une option technologique à une autre. Il est calculé par le rapport bénéfice marginal sur coût marginal exprimé en pourcentage de la façon suivante :

$$
T M R=\left[\frac{\text { Bénéfice marginal }(B M)}{\text { Coût marginal }(C M)}\right] \times 100
$$

\section{I.7. Analyse des données}

Le logiciel «Excel » a utilisé pour faire les tableaux, les figures et graphiques et le logiciel «MSTAC version 5 » pour réaliser l'analyse de variance.

\section{Présentation des résultats et discussion}

\section{II.1. Présentation des résultats}

Nous allons d'abord présenter les résultats agronomiques et ensuite les résultats économiques.

\section{II.1.1. Evaluation agronomique}

Les aspects agronomiques et les détails de l'analyse de l'évaluation sont présentés en annexes.

\section{II.1.1.1.Variables mesurées en cours de cycle}

Les résultats obtenus à partir de l'analyse des données collectées sur le nombre de talles, la hauteur moyenne de la plante, le nombre de panicules en cours de cycle sont résumés dans le tableau 4 (Annexe I).

Le nombre moyen de talles par plante varie entre 17,4 et 21,6 avec une moyenne de 19,85 cependant il n'y a pas d'effet significatif de la fumure.

Le nombre de panicules par plante varie de 13,6 à 20,8 avec une moyenne de 17,4 panicules. L'analyse de variance montre un effet significatif 
de la fumure azotée sur le nombre de panicules par plante. La comparaison des moyennes au test de classement 'Plus Petite Différence Significative' (PPDS) a permis de distinguer trois (03) groupes. Un premier groupe composé des traitements $\mathrm{T}_{2}(20,8)$ et $\mathrm{T} 3$ (18) qui ont enregistré le plus grand nombre de panicules. Un deuxième groupe qui comprend seulement $\mathrm{T}_{1}(17,2)$ et un troisième constitué de $\mathrm{T}_{4}$ qui n'a donné que 13,6 panicules par plante.

Figure 1: Nombre de panicules par plante en fonction du niveau de fertilisation

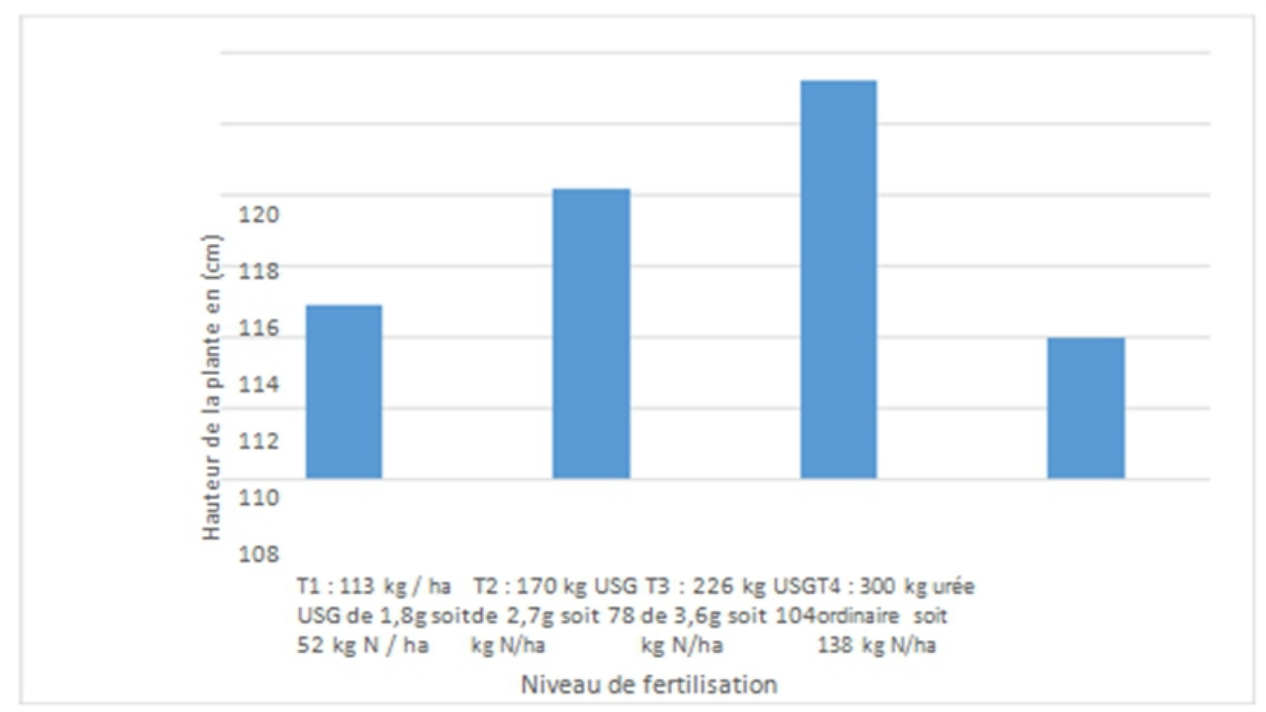

Source : auteur avec les données calculées

La hauteur des plantes à la maturité a varié suivant les traitements de 111,96 à 119,20 cm, avec une moyenne de $115 \mathrm{~cm}$. L'analyse de variance montre un effet significatif de la fumure azotée sur la hauteur de la plante. La comparaison des moyennes au test de classement PPDS permet de classer les traitements en 3 groupes : un premier groupe constitué par le traitement $\mathrm{T}_{3}$ $(119,2 \mathrm{~cm})$ avec des plantes de taille plus haute, un deuxième composé des traitements $\mathrm{T}_{2}(116,1)$ et $\mathrm{T} 1(112,8 \mathrm{~cm})$ et enfin un troisième représenté par le traitement $\mathrm{T}_{4}(112,8 \mathrm{~cm})$ avec les plantes de plus petite taille. 
Figure 2 : Hauteur de la plante en fonction du type de fumure

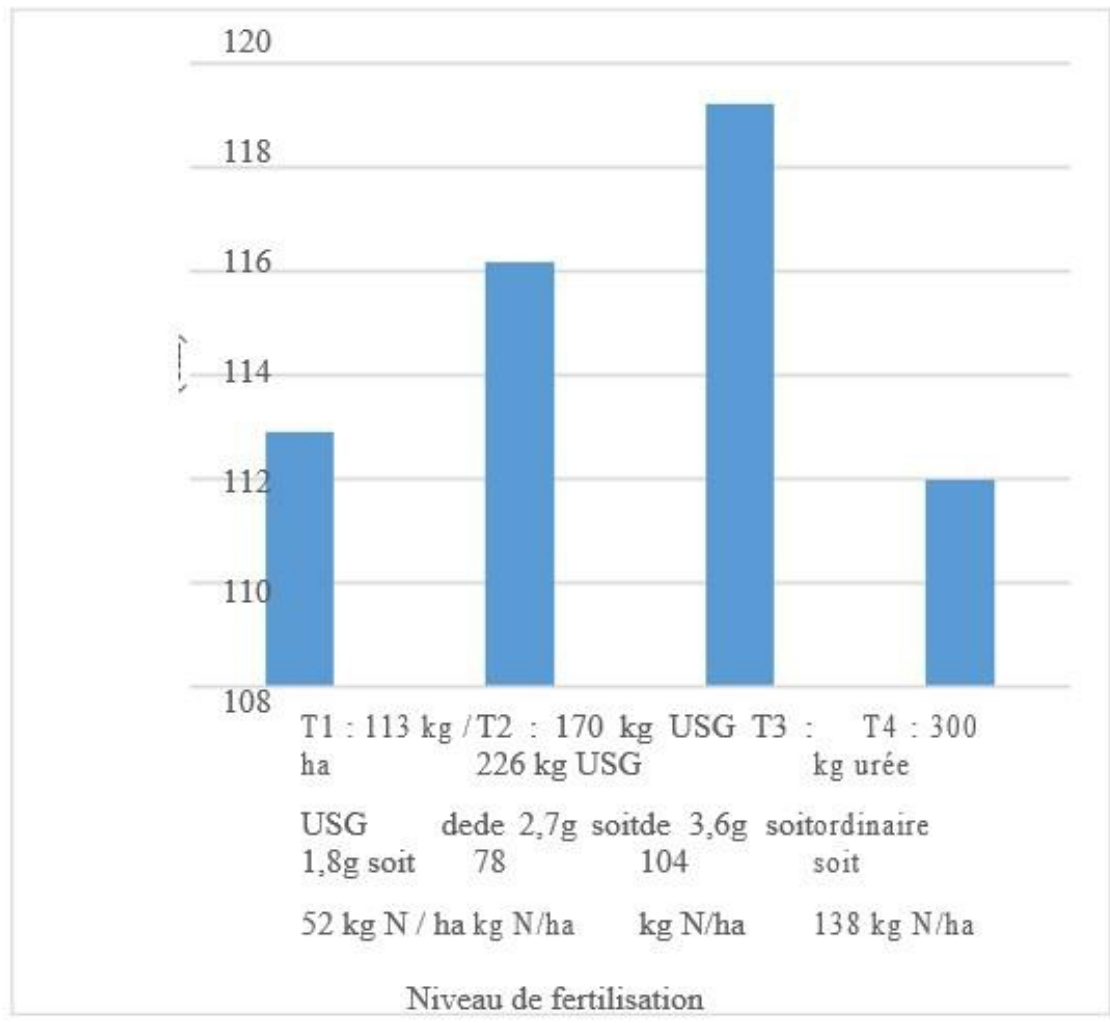

Source : auteur avec les données calculées

\section{II.1.1.2.Variables mesurées à la récolte}

Le rendement en grains paddy a varié de $5375,33 \mathrm{~kg} / \mathrm{ha}$ à 7582,66 $\mathrm{kg} / \mathrm{ha}$ avec une moyenne de $6370 \mathrm{~kg} / \mathrm{ha}$. Nous notons un effet significatif de la fumure sur le rendement en grains paddy. La comparaison des moyennes permet de distinguer deux groupes dont le premier groupe est composé des traitements $T_{3}(7582 \mathrm{~kg} / \mathrm{ha})$ et $\mathrm{T}_{2}(7082 \mathrm{~kg} / \mathrm{ha})$ qui ont donné les meilleurs rendements significativement supérieurs à ceux du deuxième groupe composé de $\mathrm{T}_{1}(5441 \mathrm{~kg} / \mathrm{ha})$ et $\mathrm{T}_{4}(5375 \mathrm{~kg} / \mathrm{ha})$ (Annexe II : Tableau 5).

Le poids de 1000 grains varie entre 21,6 et $23,7 \mathrm{~g}$ avec une moyenne de 23,10 g mais la technique de fertilisation azotée n'a pas d'influence sur ce paramètre. (Annexe II : Tableau 5).

Le rendement en paille varie entre $12875 \mathrm{~kg} / \mathrm{ha}$ et $11000 \mathrm{~kg} / \mathrm{ha}$, cependant le niveau de fumure n'a pas d'effet significatif sur cette variable. (Annexe II : Tableau 5)

L'indice de récolte est compris entre 0,54 et 0,62 avec une moyenne de 0,58 . Les résultats montrent qu'il n'y a pas d'effet significatif de la 
fertilisation azotée entre ces deux techniques de fertilisation azotée pour ce paramètre. (Annexe III : Tableau 6)

L'efficience de l'azote est la capacité qu'a la plante de riz de transformer la quantité d'urée apportée en quantité de paddy. L'efficience de l'azote a varié de 17,47 à $50,23 \mathrm{~kg}$ paddy $/ \mathrm{kg} \mathrm{N}$ avec une moyenne de $24,50 \mathrm{~kg}$ paddy $/ \mathrm{kg} \mathrm{N}$. Le niveau de fertilisation influence significativement $(\mathrm{Pr}<$ 0,0001) l'efficience de l'azote avec une supériorité du placement profond d'urée comparé à la pratique d'épandage à la volée de l'urée. On note également que l'efficience augmente avec la diminution de la taille des super granules dans le cas du placement profond d'urée . La comparaison des niveaux de fertilisation permet de distinguer trois groupes. Le premier groupe est constitué $\mathrm{T}_{1}(113 \mathrm{~kg} /$ ha USG de 1,8 g) qui révèle l'efficience la plus élevée avec $50,23 \mathrm{~kg}$ paddy $/ \mathrm{kg} \mathrm{N}$, le deuxième groupe est composé de $170 \mathrm{~kg} / \mathrm{ha}$ USG de $2,7 \mathrm{~g}\left(\mathrm{~T}_{2}\right)$ et $226 \mathrm{~kg} / \mathrm{ha}$ USG de 3,6 $\mathrm{g}\left(\mathrm{T}_{3}\right)$ avec, respectivement, 39,32 et $31,50 \mathrm{~kg}$ paddy $/ \mathrm{kg} \mathrm{N}$ et enfin le troisième groupe est constitué de $300 \mathrm{~kg} / \mathrm{ha}$ urée ordinaire (T4) $(17,41 \mathrm{~kg}$ paddy $/ \mathrm{kg} \mathrm{N})$ qui a enregistré l'efficience la plus faible (fig 3). (Annexe III : Tableau 6).

Figure 3 : Rendement de paddy en fonction du niveau de fertilisation

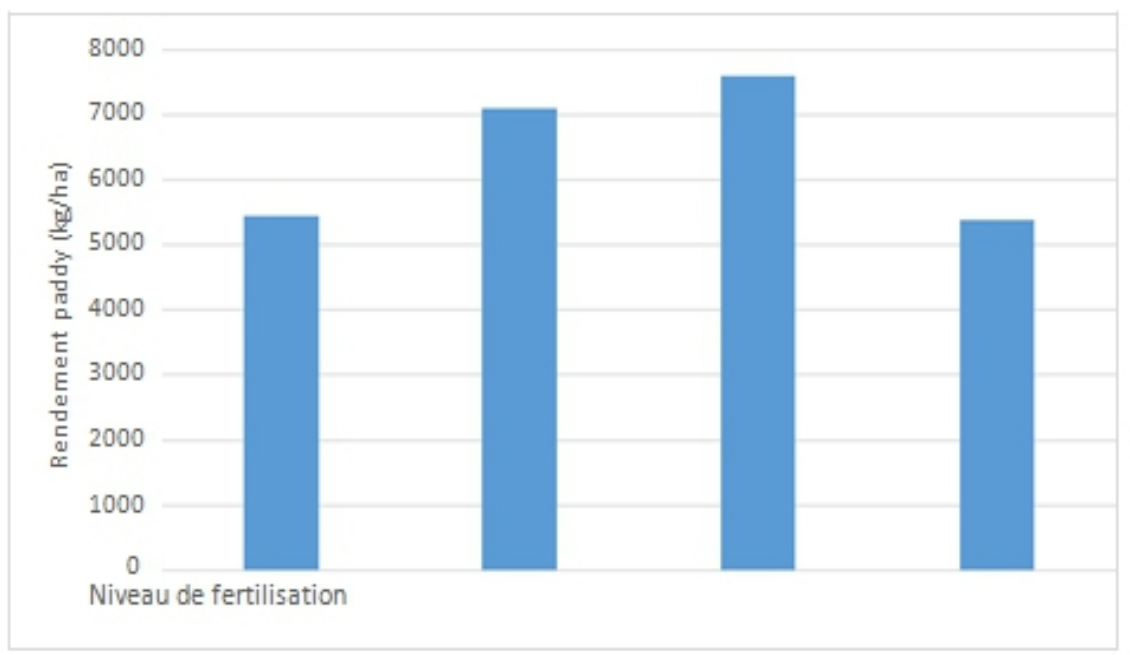

Source : auteur avec les données calculées

\section{II.1.2. Evaluation économique}

L'évaluation économique nous permet de déterminer les niveaux de fertilisation les plus intéressants qui seront recommandés aux producteurs. Nous allons commencer par l'élaboration de budgets partiels suivie d'une analyse de dominance et terminer par la détermination du taux marginal de rentabilité. 


\section{II.1.2.1.Budgets partiels}

L'approche par budgets partiels est utile pour analyser des expérimentations où l'on compare des pratiques alternatives d'une technique. Dans notre recherche, nous évaluons le gain net du changement en allant de la pratique d'épandage à la volée de l'urée au placement profond de super granules d'urée. Les résultats issus de cette analyse sont présentés dans le tableau 1:

Tableau 1: Budget partiel selon les niveaux de fertilisation en F CFA

\begin{tabular}{|c|c|c|c|c|}
\hline \multirow[t]{2}{*}{ LIBELLE } & \multicolumn{3}{|c|}{ PPU } & \multirow{2}{*}{$\begin{array}{l}\text { PP: urée } \\
300 \text { kg }\end{array}$} \\
\hline & $\overline{1,8 \mathrm{~g}}$ & $2,7 \mathrm{~g}$ & $3,6 \mathrm{~g}$ & \\
\hline \multicolumn{5}{|l|}{ Coût des intrants } \\
\hline Semences & 15000 & 15000 & 15000 & 15000 \\
\hline DAP & 17600 & 17600 & 17600 & 17600 \\
\hline Urée & & & & 47850 \\
\hline super granules d'urée ( USG) & 19831,5 & 29835 & 39663 & \\
\hline Malathion & 10000 & 10000 & 10000 & 10000 \\
\hline Sacs & 10900 & 14200 & 15200 & 10800 \\
\hline 1. Sous total coûts des Intrants & 73331,5 & 86635 & 97463 & 101250 \\
\hline \multicolumn{5}{|l|}{ Coût opérationnel } \\
\hline Confection diguettes/Planage & 7500 & 7500 & 7500 & 7500 \\
\hline Offsetage & 27000 & 27000 & 27000 & 27000 \\
\hline Gestion des irrigations & 12000 & 12000 & 12000 & 12000 \\
\hline Coût hydraulique & 80000 & 80000 & 80000 & 80000 \\
\hline redevance OMVS & 5500 & 5500 & 5500 & 5500 \\
\hline Repiquage & 30000 & 30000 & 30000 & 30000 \\
\hline épandage DAP & 1500 & 1500 & 1500 & 1500 \\
\hline épandage d'urée & & & & 3000 \\
\hline enfouissement USG & 22500 & 22500 & 22500 & \\
\hline Sarclages & 37500 & 37500 & 37500 & 45000 \\
\hline Traitement phytosanitaire & 1500 & 1500 & 1500 & 1500 \\
\hline Récolte mécanique & 66000 & 66000 & 66000 & 66000 \\
\hline Transport & 19043,5 & 24787 & 26537 & 18812,5 \\
\hline 2. Sous total coûts Opérationnels & 310043,5 & 315787 & 317537 & 297812,5 \\
\hline 3. Coût Variable de Production FCFA : CV=(1)+(2) & 383375 & 402422 & 415000 & 399062,5 \\
\hline 4. Rendement (kg/ha) & 5441 & 7082 & 7582 & 5375 \\
\hline 5. Valeur de la production en FCFA: VP(4)*135 & 734535 & 956070 & 1023570 & 725625 \\
\hline 6. Bénéfice Net en FCFA $(B N)=(5)-(3)$ & 351160 & 553648 & 608570 & 326562,5 \\
\hline
\end{tabular}

Source : auteur avec les données collectées et calculées 
L'examen de ce tableau montre que les niveaux de fertilisation avec la technologie du placement profond de l'urée donnent des bénéfices nets de 351160FCFA, 553648FCFA et 608570 FCFA / ha plus importants que la technique d'épandage de l'urée à la volée dont le bénéfice net est de 326563 F CFA / ha.

\section{II.1.2.2. Analyse de Dominance}

Le tableau 2 (Statut du niveau de fertilisation) ci-dessous nous montre que la fertilisation azotée à base d'urée épandue à la volée est une option technologique dominée qui n'est pas économiquement intéressante et doit être écartée après l'analyse.

Tableau 2: Statut du niveau de fertilisation

\begin{tabular}{cccc}
\hline Traitements & CV & BN & Statut du Traitement \\
\hline $\mathrm{T}_{1}$ & 383375 & 351160 & \\
$\mathrm{~T}_{4}$ & 399062,5 & 326562,5 & Dominé \\
$\mathrm{T}_{2}$ & 402422 & 553648 & Supérieur \\
$\mathrm{T}_{3}$ & 415000 & 608570 & Supérieur \\
\hline
\end{tabular}

Source: Auteur avec les données calculées

\section{II.1.2.3. Analyse marginale}

Le bénéfice net marginal, le coût variable marginal et le taux marginal de chaque option technologique (niveaux de fertilisation) supérieure sont calculés.

(7) Bénéfice Marginal de $T_{2}=$ Bénéfice Net de $T_{2}-$ Bénéfice Net de $T_{1}$;

(8) Bénéfice Marginal de $T_{3}=$ Bénéfice Net de $T_{3}$-Bénéfice Net de $T_{2}$;

(9) Coût Marginal (CM) de $T_{2}=$ Coût Variable $(C V)$ de $T_{2}-$ Coût variable (CV) de $T_{1}$;

(10) Coût Marginal de $T_{3}=$ Coût Variable de $T_{3}$-Cô̂t variable de $T_{2}$;

(11) Taux Marginal de Rentabilité(TMR) = [Bénéfice marginal $(B M) /$ Coût marginal (CM)] x 100

Tableau 3: Taux marginal de rentabilité

\begin{tabular}{cccccc}
\hline Traitements & CV & CM & BN & BM & TMR \\
\hline $\mathrm{T}_{1}$ & 383375 & & 351159 & 20248 & \\
$\mathrm{~T}_{2}$ & 402422 & 19047 & 553648 & 9 & $1063 \%$ \\
$\mathrm{~T}_{3}$ & 415000 & 12578 & 608570 & 54922 & $437 \%$ \\
& & & & & \\
\hline
\end{tabular}

Source: nos données 
Le tableau 3 ci-dessus montre que le taux marginal de rentabilité de $1063 \%$ allant du niveau de fertilisation actuel de $\mathrm{T}_{1}(113 \mathrm{~kg} / \mathrm{ha}$ super granules d'urée de $1,8 \mathrm{~g})$ au niveau de fertilisation de $\mathrm{T}_{2}(170 \mathrm{~kg} / \mathrm{ha}$ super granules d'urée de $2,7 \mathrm{~g})$ est plus élevé que celui (437\%) obtenu allant du niveau de fertilisation $\mathrm{T}_{2}(170 \mathrm{~kg} /$ ha super granules d'urée de $2,7 \mathrm{~g})$ au niveau de fertilisation $\mathrm{T}_{3}(226 \mathrm{~kg}$ / ha super granules d'urée de 3,6 g). Autrement dit en quittant $\mathrm{T}_{1}$ pour adopter $\mathrm{T}_{2}$, sur $100 \mathrm{~F}$ CFA investis, le producteur gagne au-delà de cet investissement initial un revenu additionnel de 1063 FCFA. Si le producteur décide de passer à $\mathrm{T}_{3}$ alors qu'il se situait déjà à $\mathrm{T}_{2}$, il aurait en plus des 1063 F CFA mentionnés un revenu additionnel de 437 FCFA sur 100 FCFA investis.

\section{II.2. Discussion}

Dans cette étude, nous avons évalué les performances agronomiques et économiques de la technologie du placement profond de l'urée super granulé (PPU) avec trois niveaux de fertilisations. Les résultats obtenus ont montré un effet significatif du PPU sur la plupart des caractères agromorphologiques étudiés (le nombre de panicules, la hauteur de la plante, le rendement paddy et l'efficience de l'azote). Cependant, il n'y a pas d'effet significatif sur le nombre de talles, le poids de 1000 grains, le poids de pailles et l'indice de récolte

Pour le nombre de talles, nos résultats sont différents de ceux obtenus par Ndiaye et al. (2010) qui ont trouvé que le nombre de talles est significativement influencé par la dose d'azote. Cette différence pourrait s'expliquer par les quantités d'azote quasi-égales, absorbées par la plante durant cette phase de culture pour ces deux techniques de fertilisation azotée. En effet, si on considère un taux de recouvrement de $30 \%$ avec l'épandage à la volée de $300 \mathrm{~kg} /$ ha d'urée, on pourrait obtenir $41,4 \mathrm{~kg} \mathrm{~N} /$ ha mis à la disposition de la plante, comparable à $35,6 \mathrm{~kg}$ N/ha avec le placement profond de $113 \mathrm{~kg}$ de super granules d'urée, pour un taux de recouvrement de $70 \%$. La faible différence entre ces deux valeurs pourrait expliquer l'absence d'effet significatif entre les deux techniques d'application de l'urée. Toutefois, il est important de noter que l'azote permet d'obtenir un bon tallage qui ne tient compte ni de la dose ni de la technique d'application

Pour le nombre de panicules par plante, l'analyse de variance montre un effet significatif de la fumure azotée. Des résultats similaires ont été obtenus au Bangladesh par Uddin et al. (2005) qui ont mis en évidence une variabilité du nombre de panicules en fonction de la source d'azote et par Asghari et al. (2006) qui ont rapporté que la dose d'azote augmente significativement le nombre de panicules par unité de surface. Les résultats de cette étude sont corroborés par ceux de Ndiaye et al. (2010) qui ont trouvé que le nombre de panicules est significativement influencé par la dose et la 
forme d'azote. En général, la fertilisation azotée, sous forme de super granules d'urée, procure un nombre plus élevé de panicules que celui obtenu avec la pratique habituelle d'épandage à la volée de l'urée.

Le rendement en paddy est significativement influencé par la forme d'azote et la taille des super granules d'urée. Nos résultats renforcent ceux de Ndiaye et al. (2010) qui ont montré que le placement profond de l'urée, avec l'utilisation de super granules d'urée, procure une plus-value de 1,12 tonne soit $21 \%$, par rapport à la pratique d'épandage à la volée. Des résultats similaires sont obtenus sur la culture du riz en Inde et au Bangladesh (Bhardwaj \& Singh, 1993) et au Burkina Faso (Yameogo, 2009 ). Le rendement augmente avec l'accroissement de la dose d'azote car le placement des granules de 3,6 et 2,7 g USG induit des plus-values respectives de 2207 $\mathrm{kg} / \mathrm{ha}(41 \%)$ et $1707 \mathrm{~kg} / \mathrm{ha}(31,7 \%)$ par rapport à la pratique d'épandage à la volée de l'urée. Selon Ndiaye et al. (2010), la maturité est significativement plus précoce avec le placement profond de l'urée qu'avec l'épandage de l'urée à la volée.

Cette étude a révélé une différence hautement significative $(\operatorname{Pr}<0,0001)$ de l'efficience de l'azote entre ces deux techniques de fertilisation azotée. En effet, l'efficience augmente avec la technique de fertilisation mais également avec la taille des super granules d'urée. La bonne performance du placement profond de l'urée pourrait s'expliquer par une meilleure utilisation d'azote par le riz à partir du pool $\mathrm{N}$ du sol enrichi par l'urée sous forme de super granules apportés à 5-15 cm de profondeur (Obcemea et al., 1984, De Datta, 1987) et par une meilleure valorisation de l'azote qui a permis de minimiser les pertes d'azote enregistrées dans le cas de l'épandage à la volée de l'urée (De Datta et al., 1989, Bhardwaj \& Singh, 1993).

Les bénéfices nets générés avec la technologie du placement profond de l'urée sont plus importants que ceux obtenus avec la pratique de l'épandage à la volée de l'urée. Ce résultat confirme celui d'IFDC (2010) qui a trouvé que le placement profond de l'urée donne des bénéfices nets plus importants que la pratique habituelle d'épandage à la volée de l'urée. La différence observée entre les bénéfices nets résulte d'une part, de la réduction considérable du coût de l'engrais pour les niveaux de fertilisation avec la technologie du placement profond de l'urée et d'autre part, du niveau de rendement plus élevé, particulièrement avec l'enfouissement des super granules d'urée de 2,7 g et 3,6 g. Les résultats ont montré que les coûts opérationnels de la technologie du placement profond de l'urée sont plus élevés que ceux de l'épandage à la volée de l'urée. Mais les niveaux des rendements, corrélativement aux valeurs de la production, sont suffisamment élevés pour compenser les coûts additionnels engendrés par l'opération d'enfouissement des super granules d'urée. 
La pratique courante veut que le taux marginal de rentabilité calculé soit comparé à un taux cible acceptable dans la zone pour déterminer le niveau de fertilisation optimale selon l'option du producteur. En effet, le producteur en investissant sur une nouvelle technologie souhaite recevoir une rémunération égale ou supérieure au gain qu'il réalise en plaçant son capital dans d'autres investissements. Un taux cible peut être estimé en se référant aux taux réservés pour les autres activités du producteur. Au Sénégal, si on tient compte des taux d'intérêt payés sur l'argent emprunté pour l'achat des vivres en période de soudure, un taux cible de $100 \%$ pourrait être retenu (Crawford et Kamuanga, 1991).

Les taux marginaux de rentabilité obtenus sur les niveaux de fertilisation correspondant à $\mathrm{T}_{3}$ et $\mathrm{T}_{2}$ sont supérieurs à ce taux cible de $100 \%$. Par conséquent, le producteur pourra les adopter car ils sont suffisamment rentables. Des résultats similaires ont été trouvés par Ndiaye et Sidibé (1999) sur le maïs dans la zone centre-sud du Bassin arachidier du Sénégal. Les performances économiques des niveaux de fertilisation $T_{2}$ et $T_{3}$ sont en adéquation avec celles trouvées à partir de l'analyse agronomique des meilleurs rendements de $7582 \mathrm{~kg} /$ ha pour $\mathrm{T}_{3}$ et $7082 \mathrm{~kg} /$ ha pour $\mathrm{T}_{2}$.

\section{Conclusion}

L'objectif général de cette étude est de contribuer à l'amélioration de la productivité du riz. Nous avons utilisé un dispositif en blocs complets randomisés avec quatre traitements (T1, T2, T3 et T4). La variété de riz utilisée est Sahel 177 et le fertilisant utilisé est l'Urée Super Granulée (USG).Deux évaluation agronomique et économique ont été faites.

La fumure azotée a un effet significatif sur le nombre de panicules par plante. La comparaison des moyennes a permis de distinguer trois (03) groupes. Un premier groupe est composé des traitements T2 $(20,8)$ et T3 (18) qui ont enregistré le plus grand nombre de panicules suivi d'un deuxième groupe constitué de T1 $(17,2)$ et un dernier groupe qui est T4 avec 13,6 panicules par plante. Elle influence aussi la hauteur de la plante avec trois groupes dont le premier est T3 $(119,2 \mathrm{~cm})$, le deuxième est T2 $(116,1)$ et T1 $(112,8 \mathrm{~cm})$ et le troisième est $\mathrm{T} 4(112,8 \mathrm{~cm})$ avec des plantes de plus petite taille. En outre, elle a un effet significatif sur le rendement en grains paddy avec deux groupes. Le premier est T3 $(7582 \mathrm{~kg} / \mathrm{ha})$ et T2 $(7082 \mathrm{~kg} / \mathrm{ha})$ qui ont donné les meilleurs rendements et le deuxième est $\mathrm{T} 1$ et $\mathrm{T} 4$. Le niveau de fertilisation influence significativement l'efficience de l'azote avec une supériorité du placement profond d'urée comparée à la pratique d'épandage à la volée de l'urée. Nous notons également que l'efficience augmente avec la diminution de la taille des super granules dans le cas du placement profond d'urée. Le classement est le suivant T1 (113 kg / ha USG de 1,8 g) ; T2 (170 $\mathrm{kg} /$ ha USG de $2,7 \mathrm{~g}$ ), T3 (226 kg /ha USG de 3,6 g) et enfin le troisième 
groupe constitué $300 \mathrm{~kg} / \mathrm{ha}$ urée ordinaire (T4) $(17,41 \mathrm{~kg}$ paddy/kg N) a enregistré l'efficience la plus faible.

Les niveaux de fertilisation avec la technologie du placement profond d'urée ont donné des bénéfices nets de 351160 à 608570 FCFA / ha plus importants que ceux de 326563 F CFA / ha enregistrés avec l'épandage de l'urée à la volée La fertilisation azotée à base d'urée épandue à la volée est une option technologique dominée qui n'est pas économiquement intéressante. La fertilisation azotée avec la technologie du placement profond d'urée permet d'obtenir des rendements plus élevés que ceux enregistrés avec l'épandage à la volée de l'urée. Cette performance est plus marquée lorsque le placement profond d'urée est pratiqué avec l'enfouissement de super granules d'urée de poids supérieur à $2,7 \mathrm{~g}$.

Nos résultats nous ont permis de recommander les niveaux de fertilisation correspondant à $\mathrm{T} 3$ et $\mathrm{T} 2$ qui sont suffisamment rentables pour être adoptés par le producteur. Les performances économiques des niveaux de fertilisation T3 et T2 sont en adéquation avec celles trouvées dans 1'analyse agronomique où les rendements obtenus sont meilleurs avec $7582 \mathrm{~kg} / \mathrm{ha}$ pour T3 et $7082 \mathrm{~kg} /$ ha pour T2.

Pour généraliser les résultats, il nous semble important de mener cette expérimentation dans d'autres conditions pédoclimatiques différentes de celles de cette recherche. En outre, il faut comparer le taux marginal calculé à un taux cible évalué à partir d'enquêtes dans les zones d'études.

\section{References:}

1. Africa Rice. (2011). Manuel Pratique de Riziculture Irriguée dans la Vallée du Fleuve Sénégal.Version 2, Février 2011. 118p.

2. Africa Rice, document non daté. Fiches Descriptives des variétés de riz homologuées au Sénégal entre 1994 et 2009.

3. ANSD. (2014). Annuaire du Commerce extérieur 2014.

4. Bhardwaj, A. K., \& Singh Y. (1993). Increasing nitrogen use efficiency through modified urea materials in mollisols in flooded rice. An agric .Res, 14(4) 448-451.

5. Crawford, E. et Kamunga, M. (1991). Analyse économique des essais agronomiques pour la formulation des recommandations aux paysans, Institut Sénégalais de Recherches Agricoles, Etude et Document, Vol 4(7), 30p.

6. De Datta, S.K. ( 1987). Nitrogen transformation processesin relation to improved cultural practices for lowland rice. Plant Soil, 100, 4769.

7. De Datta, S.K., Trevin, A.C.F., Freney, J.R., Obcemea, W.N., Real, J.G., \& Simpson,J.R. 
(1989). Measuring nitrogen losses from lowland rice using bulk aerodynamic and nitrogen-15 balance methods. Soil Sci. Am. J. (53), $1275-1281$.

8. IFDC. (2013). Le Placement Profond de l'Engrais, bulletin d'information (1).

9. ISRA. (2011). Recherche d'adaptation de la technologie du placement profond de l'urée super-granulée (USG) dans la vallée du fleuve Sénégal, rapport d'activités de l'hivernage 2010, 20p

10. Lacharme, M. (2001). La fertilisation minérale du riz, fascicule (06), Mémento technique de riziculture, $19 \mathrm{p}$.

11. Ndiaye, M., Diack, S., \& Fofana, B. (2010). Le placement profond de l'azote sous forme d'urée super granulée comme alternative pour améliorer le rendement du riz repiqué dans la vallée du fleuve Sénégal. Poster présenté à l'atelier régional organisé par CEDAO, IFDC et UEMOA, Cotonou, Benin, 13 - 15 décembre 2010. 1 p.

12. Ndiaye, M. et Sidibé, M. (1999). Recherche de formules d'engrais N$\mathrm{P}-\mathrm{K}$ économiquement rentables pour la culture du maïs pluvial. pp 281 - 295. In :B. Badu-Apraku, M.A.B. Fakorede,

13. M. Ouedraogo and R.J. Caroky (eds). Impact, Challenge and Prospects of maize Research and Development in West and Central Africa. Proceedings of a regional Maize Workshop, IITA - Cotonou, Benin Republic, 4 - 7 may, 1999. WECAMAN / IITA.

14. Obcemea, W.N., DeDatta, S.K., \& Broadhent, F.B. (1984). Movement and distribution of fertilizer nitrogen as affected by depth of placement in wetland rice. Fert. Res 5,125-148.

15. Prasad, R., Singh, S., Prasad, M., \& Thomas, J. (1982). In creased efficiency of fertilizer nitrogen applied to rice through urea super granules. Indian J. Agric. Sci., 59 (3), 154-156.

16. PNAR. (2009). Programme National d'Autosuffisance en Riz : stratégie nationale de développement de la riziculture ; $25 \mathrm{p}$.

17. PRACAS. (2014). Programme de Renforcement et d'Accélération de la Cadence de l'Agriculture Sénégalaise: stratégie de mise en œuvre du volet riz ; 48p.

18. Uddin, M. M., Eunus, M., Mannan, M.A., \& Ahmed, F.M. (2005). Effect of urea super granule on boro rice. Bangladesh J. Agric. And Environ. 1(1), 1-6.

19. Yameogo (2009). Contribution des granules d'urée dans l'amélioration des rendements. Cas de la Vallée du Kou au Burkina Faso. Mémoire d'ingénieur agronome, Université polytechnique, Bobo-Dioulasso, 40p. 


\section{ANNEXES}

Annexe I : Tableau 4: Nombre de talles, de panicules et hauteur de la plante

\begin{tabular}{|c|c|c|c|}
\hline Traitements & $\begin{array}{l}\text { Nombre } \\
\text { moyen de } \\
\text { talles / plante }\end{array}$ & $\begin{array}{l}\text { Nombre moyen de } \\
\text { panicules / plante }\end{array}$ & $\begin{array}{l}\text { Hauteur moyenne } \\
\text { de la plante }(\mathrm{cm})\end{array}$ \\
\hline $\begin{array}{l}\text { T1: } 113 \mathrm{~kg} \text { USG de 1,8g } \\
\text { soit } 52 \mathrm{~kg} \mathrm{~N} / \mathrm{ha}\end{array}$ & 19,4 & 17,2 & 112,8 \\
\hline $\begin{array}{l}\mathrm{T} 2: 170 \mathrm{~kg} \text { USG de } 2,7 \mathrm{~g} \\
\text { soit } 78 \mathrm{~kg} \mathrm{~N} / \mathrm{ha}\end{array}$ & 21,6 & 20,8 & 116,1 \\
\hline $\begin{array}{l}\text { T3 : } 226 \mathrm{~kg} \text { USG de } 3,6 \mathrm{~g} \\
\text { soit } 104 \mathrm{~kg} \mathrm{~N} / \mathrm{ha}\end{array}$ & 20,9 & 18 & 119,2 \\
\hline $\begin{array}{l}\text { T4 :300 kg urée ordinaire } \\
\text { soit } 138 \mathrm{~kg} \mathrm{~N} / \mathrm{ha}\end{array}$ & 17,4 & 13,6 & 111,9 \\
\hline $\begin{array}{l}\text { Moyenne Générale } \\
\text { CV }(\%)\end{array}$ & $\begin{array}{l}19,8 \\
12,2\end{array}$ & $\begin{array}{l}17,4 \\
12,2\end{array}$ & $\begin{array}{l}115 \\
1,75\end{array}$ \\
\hline $\begin{array}{l}\text { Test de Signification (au } \\
\text { seuil de } \alpha=5 \% \text {.) }\end{array}$ & NS & S & $S$ \\
\hline
\end{tabular}

NS : Non significatif ; $\mathrm{S}$ : significatif ; CV : Coefficient de Variation.

Annexe II : Tableau 5: Rendements en paddy, en pailles et en poids de 1000 grains

\begin{tabular}{llll}
\hline Traitements & $\begin{array}{l}\text { Rendement de } \\
\text { grains paddy } \\
\text { (kg/ha) }\end{array}$ & $\begin{array}{l}\text { Rendement en } \\
\text { pailles } \\
(\mathbf{k g} / \mathbf{h a})\end{array}$ & $\begin{array}{l}\text { Poids de } \\
\mathbf{1 0 0 0} \text { grains } \\
(\mathbf{g})\end{array}$ \\
\hline T1: $113 \mathrm{~kg}$ USG de 1,8g soit $52 \mathrm{~kg} \mathrm{~N} / \mathrm{ha}$ & 5441 & 11500 & 23,503 \\
& 7082 & 12625 & 23,790 \\
T2 $: 170 \mathrm{~kg}$ USG de 2,7g soit $78 \mathrm{~kg} \mathrm{~N} / \mathrm{ha}$ & 7582 & 12875 & 23,533 \\
& 5373 & 11000 & 21,600 \\
T3 $: 226 \mathrm{~kg}$ USG de 3,6g soit $104 \mathrm{~kg} \mathrm{~N} / \mathrm{ha}$ & 6370 & 12000 & 23,107 \\
& 10,27 & 6,87 & 3,71 \\
T4 $: 300 \mathrm{~kg}$ urée ordinaire soit $138 \mathrm{~kg} \mathrm{~N} / \mathrm{ha}$ & & & \\
Moyenne Générale & & &
\end{tabular}

Test de Signification

(au seuil de $\alpha=5 \%$.)

NS : Non significatif; $\mathrm{S}$ : significatif ; CV : Coefficient de Variation. 
Annexe III : Tableau 6 : indice de récolte et efficience de l'azote

\begin{tabular}{lll}
\hline T1: $113 \mathrm{~kg}$ USG de $1,8 \mathrm{~g}$ soit $52 \mathrm{~kg} \mathrm{~N} / \mathrm{ha}$ & 0,52 & 50,23 \\
T2 : 170 kg USG de 2,7g soit 78 kg N/ha & 0,62 & 39,32 \\
T3 : 226 kg USG de 3,6g soit $104 \mathrm{~kg} \mathrm{~N} / \mathrm{ha}$ & 0,59 & 31,50 \\
T4 :300 kg urée ordinaire soit $138 \mathrm{~kg} \mathrm{~N} / \mathrm{ha}$ & 0,54 & 17,47 \\
Moyenne Générale & 0,57 & 34,50 \\
CV $(\%)$ & 2,67 & 6,89 \\
Test de Signification & & \\
(au seuil de $\alpha=5 \%$ ) & $\mathrm{NS}$ & THS
\end{tabular}

NS : Non significatif ; CV : Coefficient de Variation ; PPDS : Plus petite différence significative 